\title{
Strange diffusion
}

\author{
R.Balescu \\ Association Euratom - Etat Belge pour la Fusion, \\ Université Libre de Bruxelles, CP 231, \\ Campus Plaine, Bd. du Triomphe, \\ 1050 Bruxelles, Belgium
}

Received March 25, 1998

\begin{abstract}
Strange diffusion is defined as a process in which the mean square displacement of a randomly walking test particle behaves asymptotically as $\left\langle x^{2}(t)\right\rangle \sim t^{\alpha}$, with $\alpha \neq 1$. A brief review of the properties of such processes is presented, stressing their deep difference from the corresponding normal diffusive evolution. A number of examples are discussed, including diffusion of charged particles in a fluctuating magnetic field, continuous time random walks and transport in chaotic Hamiltonian systems.
\end{abstract}

Key words: strange diffusion, random process, chaos

PACS: $05.40 .+j, 05.45 .+b, 83.50 . W$

\section{The problem of transport}

Whenever a physicist wants to study the evolution of a certain amount of matter, he is usually faced with a spatially inhomogeneous system. This means that the values of the intensive physical quantities (mass density, energy density,...) differ in different points. When left to itself (without any external constraints), the system evolves irreversibly towards homogeneity, by progressively equalizing these values. This evolution is necessarily accompanied by transport processes of mass, energy, etc. The first of these is associated with the diffusion phenomenon, the second with heat conduction, etc. (the list is not exhaustive). For simplicity, we only discuss here the diffusion phenomenon.

This process is described quantitatively by a matter flux $\boldsymbol{\Gamma}$ (number of molecules crossing, in a given direction, a unit area per unit time). The existence of a non-vanishing flux is due to the inhomogeneity of the particle density $n(\mathbf{x}, t)$ (number of molecules per unit volume at point $\mathbf{x}$ and at time $t$ ), measured by the density gradient $(\partial n / \partial \mathbf{x})$ : the latter plays the role of a thermodynamic force.

The study of the diffusion process requires the establishment of a functional relation between flux $\boldsymbol{\Gamma}$ and the corresponding force $(\partial n / \partial \mathbf{x})$; such a relation is 
called a transport equation (sometimes called a phenomenological relation). ${ }^{1}$ Such a relation cannot be given a priori: it should be derived from a study of the molecular mechanisms determining the system's evolution. The identification of these mechanisms and the study of their consequences constitutes the object of the transport theory. In a simple case, when the forces are not very strong, these general transport equations can be approximated by linear relations. In our case, this relation will be written as:

$$
\boldsymbol{\Gamma}=-D \frac{\partial n}{\partial \mathbf{x}}
$$

where the diffusion coefficient $D$ is assumed to be constant. The second law of thermodynamics requires, moreover, that the sign of $D$ be definitely positive: $D \geqslant$ 0. Equation (1) expresses the celebrated Fick law.

We now appeal to the continuity equation of hydrodynamics, which expresses the conservation of the number of particles:

$$
\frac{\partial}{\partial t} n=-\frac{\partial}{\partial \mathbf{x}} \cdot \boldsymbol{\Gamma}
$$

Combining equations (1) and (2), we obtain:

$$
\frac{\partial}{\partial t} n(\mathbf{x}, t)=\frac{\partial}{\partial \mathbf{x}} \cdot D \frac{\partial}{\partial \mathbf{x}} n(\mathbf{x}, t)
$$

This is the diffusion equation, well known by all physicists (as well as mathematicians, chemists, geologists, etc.). It rules the time evolution of the density profile $n(\mathbf{x}, t)$ under the action of spatial inhomogeneity.

The diffusion equation can also be interpreted probabilistically, by defining $n(\mathbf{x}, t)$ as the probability density of finding a particle at point $\mathbf{x}$, at time $t$. From the diffusion equation it is easily shown that the average value of a particle's position, $\langle\mathbf{x}(t)\rangle$, is zero at all times if it was zero initially. On the other hand, the mean square displacement, which is non-vanishing, can be obtained from equation (3) by simple partial integrations:

$$
\begin{aligned}
\frac{\partial}{\partial t}\left\langle x^{2}(t)\right\rangle & =\int \mathrm{d} x x^{2} \frac{\partial}{\partial t} n(\mathbf{x}, t) \\
& =\int \mathrm{d} x x^{2} D \frac{\partial}{\partial \mathbf{x}} \cdot \frac{\partial}{\partial \mathbf{x}} n(\mathbf{x}, t)=2 d D
\end{aligned}
$$

where $d$ is the dimensionality of the space; hence:

$$
\frac{1}{2 d} \frac{\partial}{\partial t}\left\langle x^{2}(t)\right\rangle=D(t)
$$

Here appears an apparent paradox: the left-hand side of this equation is generally a function of time; hence, the quantity appearing in the right-hand side cannot

\footnotetext{
${ }^{1}$ In more general cases, one should derive a relation between the set of fluxes and the set of forces. This leads to a transport matrix, whose symmetry properties are studied in the celebrated work by L. Onsager. We shall not discuss these aspects here.
} 
be identified with the constant diffusion coefficient appearing in equation (3). We will call $D(t)$ the running diffusion coefficient. This simple analysis shows that the macroscopic equation (3) cannot be universally valid. It appears, however, that in numerous cases, the mean square displacement starts nonlinearly (usually as $t^{2}$ ) for short times, then tends progressively toward a linear form. As a result, the running diffusion coefficient tends, for long times, toward the positive constant $D$ (see figure 1): the latter is identified, by definition, with the macroscopic diffusion
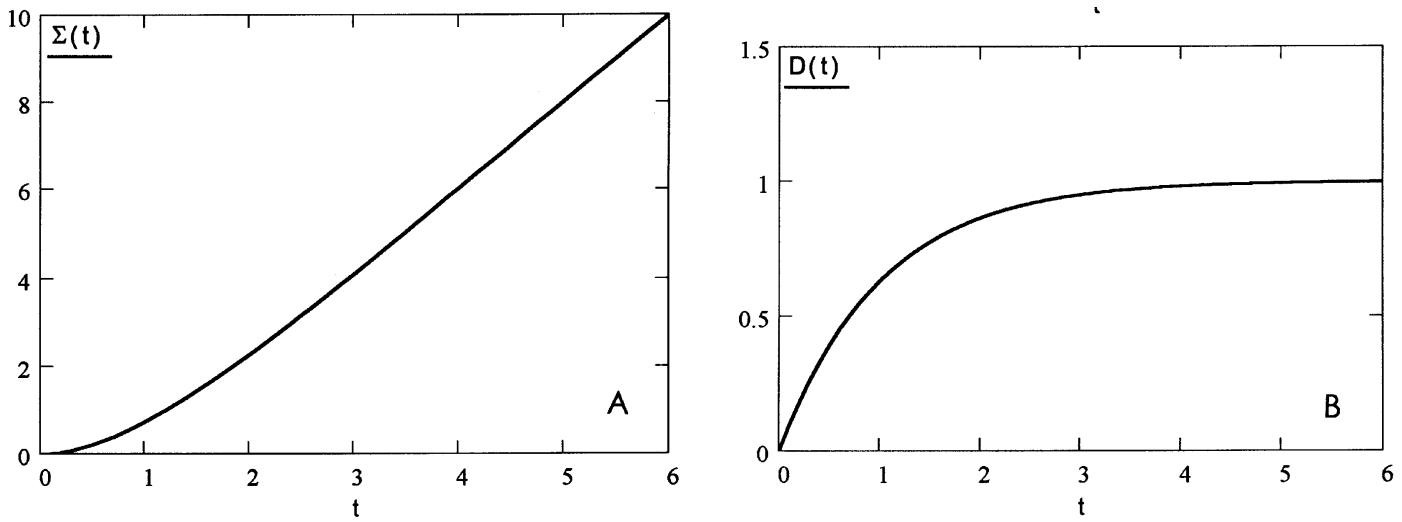

Figure 1. Mean square displacement (A) and running diffusion coefficient (B).

Diffusive regime.

coefficient:

$$
D=\lim _{t \rightarrow \infty} \frac{1}{2 d} \frac{\partial}{\partial t}\left\langle x^{2}(t)\right\rangle .
$$

The macroscopic transport equation thus appears as an asymptotic limit, valid for sufficiently long times. In the molecular transport theory, there appears, therefore, a characteristic time $\tau_{\mathrm{R}}$, such that the macroscopic laws (e.g., the emergence of a constant diffusion coefficient) become valid for times sufficiently long compared with $\tau_{\mathrm{R}}: t \gg \tau_{\mathrm{R}}$.

\section{Normal transport}

If the system under consideration were made up of strictly independent molecules, in the absence of any external field, these particles would have a uniform rectilinear motion limited only by the reflexions on the walls. Their positions would be linear functions of $t$ and their MSD would be proportional to $t^{2}$. Hence, equation (5) would yield an infinite diffusion coefficient! In order to understand the existence of a finite diffusion coefficient, we must necessarily identify one (or several) mechanism(s) that are able to slow down (on the average) the motion of the particles. A universal mechanism of this kind is provided by the particle collisions.

\section{Kinetic theory}

The most rigorous tool for the derivation of macroscopic transport processes from the basic laws of molecular dynamics is provided by non-equilibrium statisti- 
cal mechanics. In this field D. N. Zubarev was a great master: his book "Nonequilibrium statistical thermodynamics" [1] is a classic. Although the discipline was inaugurated by L. Boltzmann in 1872 [2], it only began flourishing in the second half of the 20-th century, with a large number of works of which we quote only a few [3]-[8]. Although the methods used by various authors differ in details, the main strategy is common. The theory proceeds in two steps. At the first stage it is shown that the basic Liouville equation leads asymptotically, under certain conditions, to an intermediate equation called a kinetic equation. In the second step the laws of macroscopic physics are derived from the latter (using an additional asymptotic treatment) by the averaging process.

The kinetic equation governs the time evolution of the one-particle reduced distribution function $f(\mathbf{x}, \mathbf{v} ; t)$ defined as the probability density of finding a particle at point $\mathbf{x}$ with velocity $\mathbf{v}$ at time $t .{ }^{2}$ Its general form can be written as follows (in the absence of an external field):

$$
\frac{\partial}{\partial t} f(\mathbf{x}, \mathbf{v}: t)=-\mathbf{v} \cdot \frac{\partial}{\partial \mathbf{x}} f(\mathbf{x}, \mathbf{v} ; t)+\mathcal{K}\{f\} .
$$

The first term on the right-hand side represents the free motion of the particles, the second one describes the effect of the collisions. The latter is an expression depending nonlinearly on the distribution function; its explicit form depends on the type of the system considered (gas, solid, plasma, etc.) It is out of the question to give in this short paper even an outline of the derivation or of the solution of this class of equations: these matters are treated in detail in [1]-[8]. Let us just say that, as mentioned above, an asymptotic treatment of the kinetic equation (6) leads to the diffusion equation (3) with the following expression of the "classical" diffusion coefficient:

$$
D^{\mathrm{CL}}=\frac{V_{T}^{2}}{2 \nu}=\frac{k_{\mathrm{B}} T}{m \nu} .
$$

Here $V_{T}$ represents the thermal speed of the particles related to the temperature $T$ and the mass $m$ of the molecules: $V_{T}=\left(2 k_{\mathrm{B}} T / m\right)^{1 / 2}$ (where $k_{\mathrm{B}}$ is the Boltzmann constant). $\nu$ is the collision frequency (number of collisions per unit time): its form depends on the nature of the molecular interactions, as well as of the temperature $T$ and of the density $n$ of the environment. Its specific expression is derived from the kinetic equation. This quantity defines the characteristic time mentioned above: $\tau_{\mathrm{R}}=\nu^{-1}$.

\section{Classical random walks}

Although kinetic equations are the starting point of any rigorous transport theory, their derivation and/or their solution are often extremely difficult. It thus appeared necessary to develop alternative methods that avoid the difficulties, at the price of introducing some a priori reasonable assumptions. The necessity of such alternative approaches was also mentioned by Zubarev in chapter 4 of [1]. An

\footnotetext{
${ }^{2}$ We only discuss classical mechanical systems in this paper.
} 
extreme type of such approaches is the classical theory of random walks elaborated at the beginning of this century by Einstein [9]. In this model the dynamical laws governing the motion of the molecules are replaced by purely probabilistic rules.

More precisely, it is assumed that a particle starting at the origin performs at each instant $t=n \tau$ a step of length (and direction) $\mathbf{x}$, with the transition probability density $f(\mathbf{x})$ specified in advance. The steps thus occur at discrete times separated by intervals of length $\tau$ ( $n$ is a positive integer). If $f(\mathbf{x})$ is symmetric, the average $\langle\mathbf{x}(t)\rangle=0$, but the mean square displacement $\left\langle x^{2}(t)\right\rangle \neq 0$. It appears that the latter quantity (which measures the extent of the region visited by the particle) increases linearly in time. Combining this result with the probabilistic interpretation (5), an expression for the diffusion coefficient is obtained.

Clearly, this model is much rougher than the kinetic theory, but for complex systems it is often one of the few theoretical tools available. ${ }^{3}$ The main results obtained within this framework are summarized here. The mean square displacement grows linearly in time:

$$
\left\langle x^{2}(t)\right\rangle=2 d D t .
$$

This property defines what will be called a diffusive regime. The diffusion coefficient is obtained as:

$$
D=\frac{\left\langle\left\langle x^{2}\right\rangle\right\rangle}{\tau} .
$$

Here $\left\langle\left\langle x^{2}\right\rangle\right\rangle$ is the second moment of the transition probability, namely: $\left\langle\left\langle x^{2}\right\rangle\right\rangle=$ $\int \mathrm{d} \mathbf{x} x^{2} f(\mathbf{x})$. Clearly, the diffusion coefficient is determined by the "pseudo-dynamical law" defined by $f(\mathbf{x})$; it is automatically a positive quantity. A more detailed result is obtained in the theory of random walks: the form of the density profile $n(\mathbf{x}, t)$ :

$$
n(\mathbf{x}, t)=\frac{1}{(4 \pi D t)^{d / 2}} \exp \left[-\frac{x^{2}}{4 D t}\right] .
$$

The density profile thus has, at all times, a Gaussian form. This result is an expression of the celebrated central limit theorem of the probability theory. It is easily checked that expression (10) is a fundamental solution of the diffusion equation (3) which reduces to the delta-distribution $\delta(\mathbf{x})$ at $t=0$. The three results (8)-(10) are the signature of the normal transport (or classical transport) law. This law is applicable to a large number of "usual" situations. The "perverse" physicist, however, is eager to find exceptions...

\section{Anomalous diffusion}

In the situations discussed above the dissipative mechanism controlling diffusion consists of interparticle collisions. There exist, however, very interesting and important situations where the collisions become extremely rare: this happens, for instance, in plasmas at very high temperatures. Nevertheless, in these cases there

\footnotetext{
${ }^{3}$ An intermediate, "semidynamical" approach will be discussed below.
} 
exist transport processes characterized by finite coefficients. This observation implies that some phenomena, other than individual particle collisions, introduce the necessary dissipation. These are collective phenomena involving simultaneously a large number of particles acting synchronously. One must consider in this case the density fluctuations and/or the electromagnetic field fluctuations producing waves which exist in a large variety in plasmas. These excitations interact globally producing scattering, emission, absorption or transformation processes which introduce dissipation. When the amplitude of these excitations increases (in particular, as the result of their intrinsic instability), their nonlinear interactions may lead (through a cascade of bifurcations) to a new state, far from the equilibrium, called a turbulent state.

The problem of transport in turbulent states remains one of the most difficult problems of physics: up till now it has only partial answers in specific cases. It may be shown in certain instances that a positive diffusion coefficient may exist. In comparison with our discussion of the normal transport, we may underscore the following characteristics:

- The mean square displacement is asymptotically a linear function of time of the form (8).

- The diffusion coefficient is not of the form (7). It is not (or not only) controlled by the collision frequency $\nu$ (which is either zero or very small) but rather by the characteristics of the non-collisional dissipative processes, such as the intensity of fluctuations, the correlation lengths, etc.

- The density profile remains Gaussian (10) (at least asymptotically), hence this quantity is the solution of the diffusion equation (3).

Whenever these three conditions are combined, we speak, by definition, of an anomalous transport regime: it is a diffusive but non-collisional regime. We cannot summarize here the enormous amount of literature devoted to this problem (see, e.g., [10]). A specific example will appear below.

\section{Strange transport}

The anomalous transport regime differs from the normal one only by the dissipation mechanism controlling it (the difference is, however, not a minor one, as it results from the difficulty of establishing a rigorous theory of these processes). We now make a decisive step that will lead us to "another world". The new regime considered here is defined by the following criteria:

- The mean square displacement tends asymptotically toward the following form:

$$
\left\langle x^{2}(t)\right\rangle \underset{t \rightarrow \infty}{\Longrightarrow} A t^{\alpha}, \quad \alpha \neq 1
$$


- The coefficient $\alpha$ is called a diffusion exponent. ( $A$ is a positive dimensional constant). The present regime is thus definitely non-diffusive; if $\alpha=1$, the diffusive regime is recovered.

- The running diffusion coefficient $D(t)$ behaves asymptotically as follows:

$$
D(t) \underset{t \rightarrow \infty}{\Longrightarrow} C t^{\alpha-1}
$$

- The running diffusion coefficient $D(t)$ is controlled by non-collisional (or partially collisional) mechanisms.

- The density profile is non-Gaussian.

These features together define a strange transport regime. ${ }^{4}$ Two types of strange transport can be distinguished.

- The superdiffusive regime is characterized by the diffusion exponent $\alpha>1$. In this case, equation (12) shows that:

$$
\lim _{t \rightarrow \infty} D(t)=\infty . \quad(\text { superdiffusive })
$$

- The subdiffusive regime is characterized by the diffusion exponent $\alpha<1$. In this case,

$$
\lim _{t \rightarrow \infty} D(t)=0 . \quad \text { ( subdiffusive) }
$$

We now discuss several examples of strange transport.

\section{Diffusion of charged particles in a fluctuating magnetic field}

We consider a collection of independent charged test particles moving in the presence of an external magnetic field. The latter is a superposition of the (strong) constant field $\mathbf{B}_{0}$ directed along the $z$-axis of a cartesian reference frame, and a stationary ("frozen") fluctuating perpendicular component directed (for the convenience) along the $x$-axis, and depending on $z:{ }^{5}$

$$
\mathbf{B}(z)=B_{0}\left[\mathbf{e}_{z}+b(z) \mathbf{e}_{x}\right] .
$$

\footnotetext{
${ }^{4}$ Many authors call this regime anomalous transport. The same terminology is used by other authors in the sense defined in section 3 . We believe that it is very important to distinguish between these two completely different types of phenomena [8]. The term "strange kinetics" first appeared in an important work by Shlesinger, Zaslavsky and Klafter [11].

${ }^{5}$ In general, $b(z)$ depends on the three coordinates $x, y, z$. The assumption of the dependence on $z$ alone yields a major simplification, as it will be seen below.
} 
Here $\mathbf{e}_{x}, \mathbf{e}_{z}$ are unit vectors, $B_{0}$ is the amplitude of the unperturbed field, and $b(z)$ is statistically defined by a homogeneous, centred Gaussian stochastic process characterized by the following correlation function:

$$
\left\langle b\left(z_{1}\right) b\left(z_{1}+z\right)\right\rangle=\mathcal{B}(z)
$$

where $\mathcal{B}(z)$ is a function of $z$, given a priori. ${ }^{6}$ The following form may be chosen for the convenience:

$$
\mathcal{B}(z)=\beta^{2} \exp \left(-\frac{z^{2}}{2 \lambda^{2}}\right) .
$$

This function introduces two characteristic parameters: $\beta$ measures the intensity of fluctuations and $\lambda$ is the correlation length. This problem can be solved by a "semi-dynamical" method (see the footnote ${ }^{2}$ ).

\section{The Langevin equations}

The magnetic field lines associated with (15) obey the following purely geometric equation defining the coordinate $x(z)$ along the line, in terms of the parameter $z:^{7}$

$$
\frac{\mathrm{d} x(z)}{\mathrm{d} z}=b(z)
$$

Given that $b(z)$ is not a deterministic quantity, the "solution" $x(z)$ of equation (18) cannot take numerical values; only the averages of the functions of $x(z)$ over the ensemble of realizations of $b(z)$ have meaningful values. Equation (18) belongs to the class of stochastic differential equations first introduced by Langevin in 1908 in the theory of the Brownian motion (see: [12]). From equation (18) it is not difficult to obtain an expression of the mean square displacement of the magnetic field lines:

$$
\left\langle x^{2}(z)\right\rangle \underset{z \rightarrow \infty}{\Longrightarrow} 2 D_{\mathrm{m}} z
$$

In this problem, where $z$ plays the role of "time", we find an asymptotically diffusive behaviour. Starting from a given point on a field line and advancing in the $z$-direction, the field line becomes more and more diffusive because of the fluctuations: this is the phenomenon of magnetic diffusion which introduces the magnetic diffusion coefficient $D_{\mathrm{m}}$.

We now introduce test particles in this field. In the first approximation, if the unperturbed field $\mathbf{B}_{0}$ is sufficiently strong, the particles' position can be replaced by the position of their guiding centres which move (upon neglecting the slow drift motion) in a direction parallel to the (perturbed) field and undergo, from time to

\footnotetext{
${ }^{6}$ In a completely self-consistent theory, the particle's motion retroacts on the magnetic field, and one should use a coupled system of equations for the plasma and the magnetic field. In the present simplified model, independent particles move under the action of an independently prescribed field (for this reason they are called test particles rather than plasma particles).

${ }^{7}$ It is now seen that if the fluctuating field were to depend on the coordinate $x$, the right-hand side of equation (18) would be $b[x(z), z]$, and the equation would be highly nonlinear.
} 
time, a collision. The $z$ coordinate of the test particle is thus represented by the following equation:

$$
\frac{\mathrm{d} z(t)}{\mathrm{d} t}=v(t)
$$

The right-hand side is a random quantity modelling the action of the collisions. It is also assumed to be a stationary, centred Gaussian stochastic process characterized by the following correlation function:

$$
\left\langle v(t) v(t+\tau\rangle=\mathcal{R}^{0}(\tau)\right.
$$

where $\mathcal{R}^{0}(\tau)$ is a prescribed function:

$$
\mathcal{R}^{0}(\tau)=\frac{1}{2} V_{T}^{2} \exp (-\nu \tau)
$$

We may note that the calculation of the ("parallel") diffusion coefficient from equations (20)-(22) - which do not depend on the magnetic field! - yields exactly the classical, purely collisional diffusion coefficient $D^{\mathrm{CL}}$, equation (7).

Combining equations (18) and (20), we find the equation for the $x$-coordinate of the test particle:

$$
\frac{\mathrm{d} x(t)}{\mathrm{d} t}=b[z(t)] v(t)
$$

We now have to treat a doubly stochastic equation, involving both the collisional fluctuations and the magnetic ones: it was solved exactly in [13], [14]. The result for the mean square displacement $\left\langle\delta x^{2}(t)\right\rangle \equiv\left\langle\left[(x(t)-x(0)]^{2}\right\rangle\right.$ is a highly nonlinear function of time:

$$
\left\langle\delta x^{2}(t)\right\rangle=4 D^{\mathrm{CL}} \frac{\beta^{2}}{\gamma \nu}\left\{[1+\gamma \psi(\nu t)]^{1 / 2}-1\right\},
$$

where the function $\psi(x)=x-1+\exp (-x)$, and:

$$
\gamma=\frac{V_{T}^{2}}{\nu^{2} \lambda^{2}}=\left(\frac{\lambda_{\mathrm{mfp}}}{\lambda}\right)^{2}
$$

The parameter $\gamma$, square of the ratio of the collisional mean free path $\lambda_{\mathrm{mfp}}$ to the correlation length, is a measure of the collisionality (large $\gamma$ is equal to small collisionality). It is easily found that the mean square displacement starts like $t^{2}$ for short time, whereas asymptotically, for large $t$, it tends towards:

$$
\left\langle\delta x^{2}(t)\right\rangle \underset{t \rightarrow \infty}{\Longrightarrow} 4 D^{\mathrm{CL}} \frac{\beta^{2}}{\sqrt{\gamma \nu}} t^{1 / 2} .
$$

We thus found a typical strange, subdiffusive behaviour, as in equation (11), with $\alpha=1 / 2$. The corresponding ("perpendicular") running diffusion coefficient behaves asymptotically, as:

$$
D(t)=D^{\mathrm{CL}} \frac{\beta^{2}}{\sqrt{\gamma \nu}} t^{-1 / 2} ;
$$


it tends to zero as $t \rightarrow \infty$. This subdiffusive behaviour expresses the fact that the particles "stick" to the magnetic field lines: they move in the lateral direction only because the field lines themselves are diffusing. The mean square displacement and the running diffusion coefficient are shown in figure 2 (to be compared with figure 1).
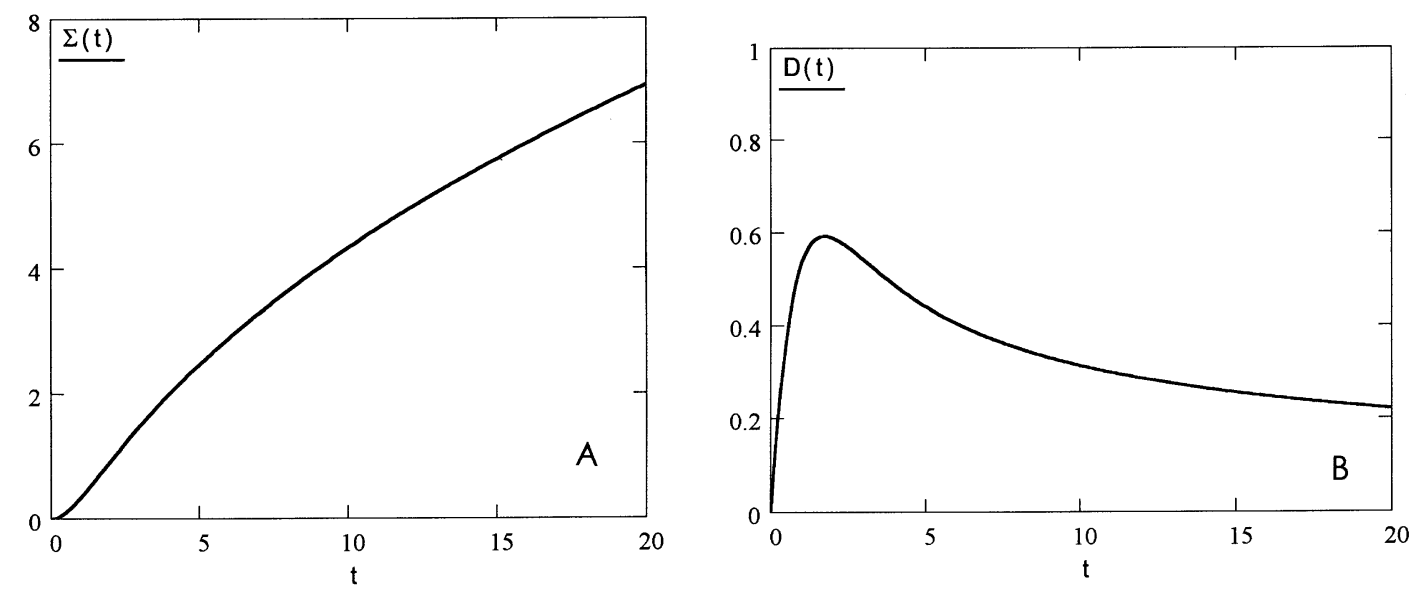

Figure 2. Mean square displacement (A) and running diffusion coefficient (B). Subdiffusive regime.

\section{The hybrid kinetic equation}

It is always possible to associate with the Langevin equations (20) and (23) a kinetic equation for the distribution function of particles in the space $(x, z)$ : $F(x, z ; t)$ :

$$
\frac{\partial F(x, z ; t)}{\partial t}+v(t) \frac{\partial F}{\partial z}+v(t) b(z) \frac{\partial F}{\partial x}=0
$$

The main difference from the ordinary kinetic equation, e.g. (6), is that the velocity is not considered as a phase space variable, but rather as a given function of $t$ in every realization, globally defined statistically by equation (21); similarly, $b(z)$ is defined statistically by equation (16). Equation (28) thus incorporates both the statistical aspect of the phase space probability distribution (as in ordinary statistical mechanics) and additional fluctuations of the coefficients. It will be called the hybrid kinetic equation [13], [8] (sometimes also "stochastic Liouville equation", a rather improper name). Although containing the same physics as the Langevin equation, the hybrid kinetic equation provides a direct access to more refined physical quantities (such as the density profile) and can treat problems for which the Langevin equation is not applicable [15].

A rather standard calculation [13], [8] leads to the derivation of an equation for the radial density profile, defined here as the average of the distribution function over the ensemble of magnetic and collisional fluctuations and integrated over the 
coordinate $z, n(x, t)=\int \mathrm{d} z\langle F(x, z ; t)\rangle$ :

$$
\frac{\partial n(x, t)}{\partial t}=\int_{0}^{t} \mathrm{~d} \theta R(\theta) \frac{\mathrm{d}^{2}}{\mathrm{~d} x^{2}} n(x, t-\theta) .
$$

This evolution equation resembles the diffusion equation (as it contains the Laplacian on the right-hand side), but differs from the latter in an essential aspect. The rate of change of $n(x, t)$ depends not only on the instantaneous state at time $t$, but on the whole history of situations between 0 and $t$. The influence of history is contained in the memory function $R(\theta)$ whose explicit expression is obtained from the hybrid kinetic equation. Equation (29) is properly called a non-Markovian diffusion equation.

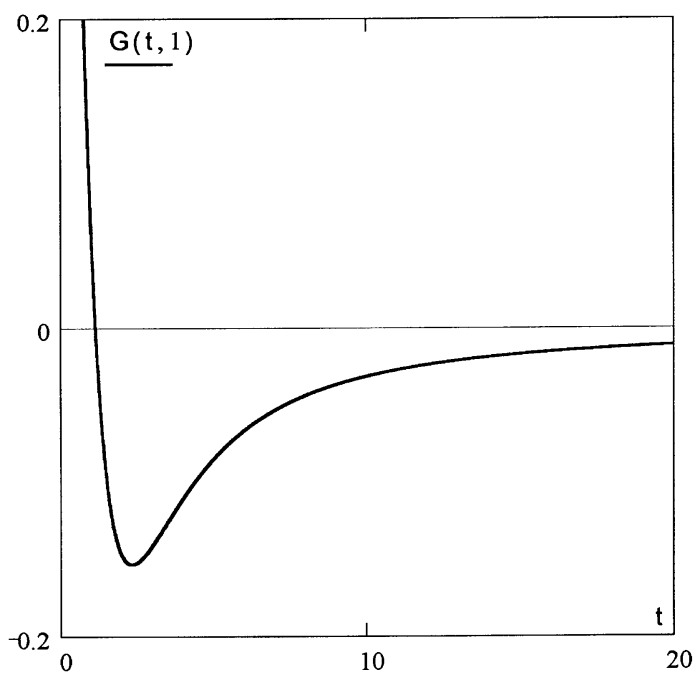

Figure 3. Memory function in the nonMarkovian diffusion equation.
Whenever the memory function decays very fast (exponentially) over a characteristic correlation time $\tau_{C}$ during which the density profile is barely changed, the retardation can be neglected, $n(x, t-\theta) \approx n(x, t)$, and the limit of integration can be extended to infinity. As the result of this asymptotic approximation (markovianization), equation (29) reduces, for $t \gg$ $\tau_{\mathrm{C}}$, to the ordinary diffusion equation (3), with the following identification of the diffusion coefficient:

$$
D=\int_{0}^{\infty} \mathrm{d} \theta R(\theta) .
$$

In our present problem, however, the situation is different. It appears that the memory function decays very slowly for long times, as a power law (figure 3):

$$
R(\theta) \sim \theta^{-3 / 2}
$$

As a result, the Markovian approximation is no longer justified; one must solve directly the non-Markovian diffusion equation. The subdiffusive regime thus leads to a deep change of the evolution law. This change is responsible, in particular, for the non-Gaussian density profile which appears as the solution of equation (29). The latter has at all times a long tail, as can be seen in figure 4 . This figure also shows the solution of equation (29), arbitrarily markovianized, with the memory function (31). The large difference between the two functions is clearly visible.

On the other hand, the running diffusion coefficient, defined as in equation (30) with an upper integration limit equal to $t$, rather than $\infty$, appears to be identical to the result derived from equation (24) for all times, and reduces to (27) for long times. [This can be immediately checked qualitatively from equation (31)]. 

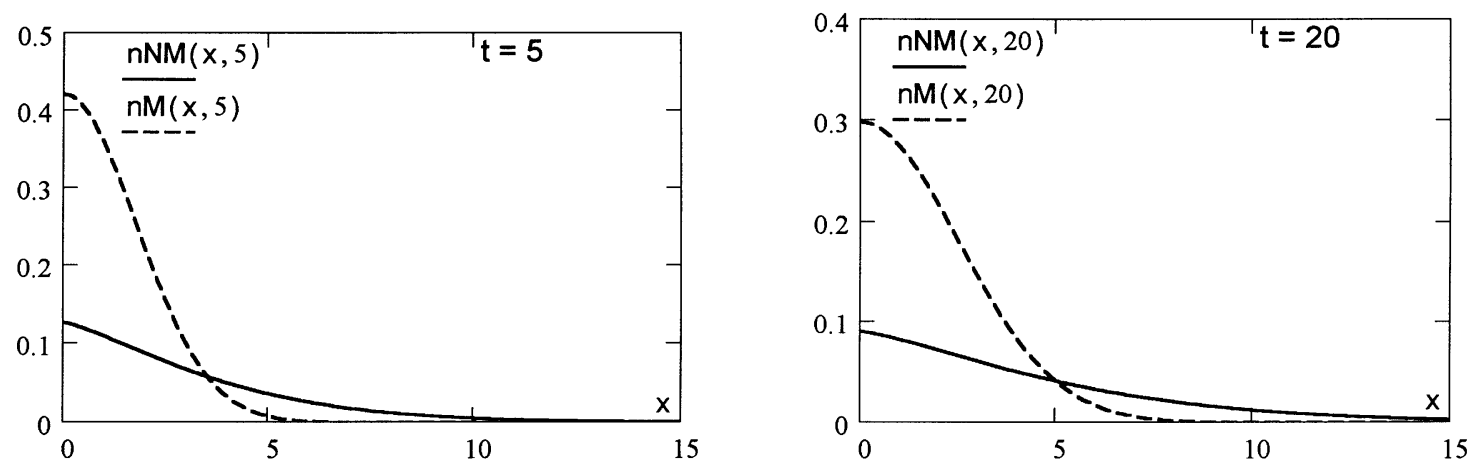

Figure 4. Subdiffusive (solid line) and markovianized (dashed line) density profiles at two different times.

\section{Continuous time random walks (CTRW)}

We now consider a remarkable generalization of the classical concept of a random walk introduced by E. Montroll and his co-workers in 1965 [16] and beautifully exposed in a review paper [17]. It is only recently that this concept began to exploit its many possibilities (e.g., [18], [11], [19], [14], [8]). In this model, a test particle performs uncorrelated steps of length and direction defined by $\mathbf{x}$, at times $t$ which are random. As this description no longer considers time as an integer multiple of an elementary quantum, but rather as a real variable, the corresponding model is called a Continuous Time Random Walk (CTRW). In order to define the problem, two functions must be specified a priori:

- $f(x)$ : the probability density of step $\mathbf{x}$ (the same object as in the classical random walk theory),

- $p(t)$ : the probability density of a waiting time of length $t$ between two successive steps.

The CTRW defined in this way is an exactly solvable problem.

This generalization of the classical random walk is very flexible; it turns out to be a remarkable tool for the study of strange transport. In particular, we may choose (in one dimension) for $f(x)$ a Gaussian distribution (at least at a large distance):

$$
f(\mathbf{x}) \sim \exp \left(-\frac{r^{2}}{2 \sigma^{2}}\right)
$$

where $r=(\mathbf{x} \cdot \mathbf{x})^{1 / 2}$, and $\sigma$ is a characteristic length. For the waiting time distribution we assume a power law decay (for large $t$ ):

$$
p(t) \sim\left(\frac{t}{\tau_{\mathrm{D}}}\right)^{-1-\alpha}, \quad 0<\alpha<1,
$$

where $\tau_{\mathrm{D}}$ is a characteristic time. This model will be called the standard longtail CTRW (SLT-CTRW). It is then shown that the system behaves according to 
the strange transport law, for which $\alpha$ is precisely the diffusion exponent. More precisely, the following results are obtained [8], [14], [17], [19]:

- The mean square displacement behaves asymptotically as follows:

$$
\left\langle x^{2}(t)\right\rangle \sim\left(\frac{t}{\tau_{\mathrm{D}}}\right)^{\alpha} .
$$

- The density profile obeys the non-Markovian diffusion equation:

$$
\frac{\partial n(\mathbf{x}, t)}{\partial t}=\int_{0}^{t} \mathrm{~d} \theta R_{\alpha}(\theta) \nabla^{2} n(\mathbf{x}, t-\theta) .
$$

- The memory function has a long-tail power law behaviour (for long times):

$$
R_{\alpha}(\theta) \sim\left(\frac{\theta}{\tau_{\mathrm{D}}}\right)^{-2+\alpha} .
$$

- The density profile [fundamental solution of equation (35)] is non-Gaussian; it reduces to a Gaussian one only when $\alpha=1$ :

$$
n(r, t)=\sigma^{-d}\left(\frac{t}{\tau_{\mathrm{D}}}\right)^{-\alpha d / 2} \exp \left(-b \xi^{2 /(2-\alpha)}\right) .
$$

- The density profile obeys the scaling law, i.e. it depends on $\mathbf{x}$ only through the similarity variable $\xi$ :

$$
\xi=\frac{r}{t^{\alpha / 2}} .
$$

These results show that the SLT-CTRW bears all the signatures of a strange diffusion evolution of the subdiffusive type. It is interesting to compare these results, for $\alpha=1 / 2$, with the exact solution of our test problem from the previous section. It appears [14] that, with some rather weak restrictions, the SLT-CTRW is an excellent model for the strange diffusion in a fluctuating magnetic field, even for predicting very refined quantities.

\section{Chaotic Hamiltonian dynamical systems}

Non-integrable dynamical systems have been very extensively studied in the past three decades, both analytically and numerically [20] - [23]. In particular, iterative maps are simple models exhibiting the complex nature of orbits of dynamical systems, especially Hamiltonian systems. A map that was used by many authors as a paradigm applicable to a variety of problems is the standard map introduced by Chirikov and Taylor in 1983 [20]. It is a two-dimensional map describing the evolution at discrete times $\nu=0,1,2, \ldots$ of the (action) variable $x_{\nu}$ and the angle variable $\theta_{\nu}$ :

$$
x_{\nu+1}=x_{\nu}-\frac{K}{2 \pi} \sin 2 \pi \theta_{\nu}, \quad \theta_{\nu+1}=\theta_{\nu}+x_{\nu+1}(\bmod 1) .
$$


$K$ is the stochasticity parameter. For small finite values of $K$, the phase portrait consists of island chains around periodic orbits surrounded by thin stochastic layers and sandwiched between KAM (Kolmogorov - Arnol'd - Moser) surfaces. As $K$ increases, the stochastic layers become thicker and the KAM surfaces are progressively destroyed. Above the critical value $K_{c}$ all KAM barriers are destroyed, and any chaotic orbit wanders up to unlimited values of $x$ : this is the state of a global chaos. We are interested here in the relation of the standard map to the transport of

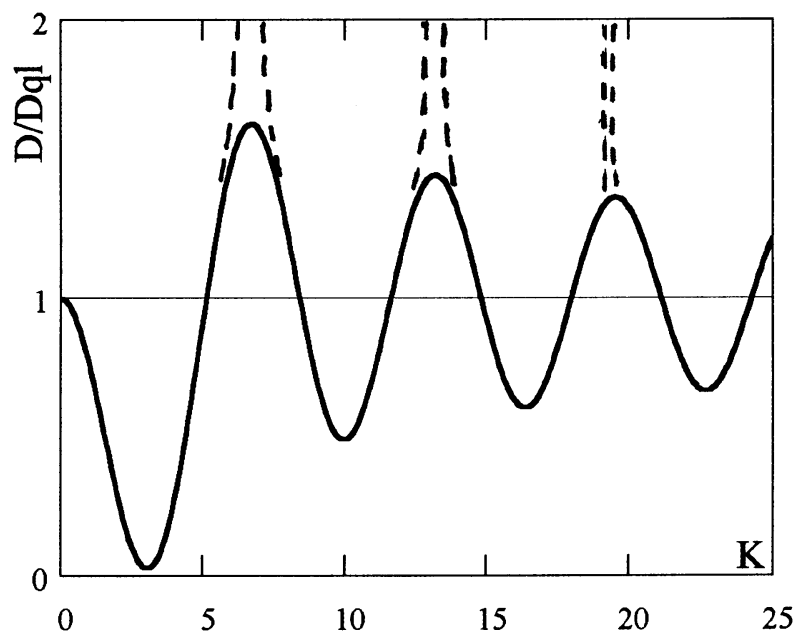

Figure 5. Diffusion coefficient vs. stochasticity parameter in the standard map: diffusive and superdiffusive regimes.

"particles" in the $x$-direction. The standard map appears to describe all types of the normal and strange diffusion regimes according to the value of the stochasticity parameter $K$.

It has been known for a long time [24] that in the limit of very large stochasticity, i.e. in the region of a global chaos, the standard map describes a typical diffusive evolution. This result has been recently derived in a very rigorous way, by using the methods of statistical mechanics, by Hasegawa and Saphir [25], [8]. It is stated precisely as follows. Consider a distribution of particles over the phase space described by the density profile $n(x, \nu)$. The following limiting domain of the parameters is defined:

$$
\frac{1}{\sqrt{K}} \ll 1, \quad \frac{x}{K} \gg 1
$$

Thus, the stochasticity parameter is very large, but simultaneously, the distances considered are very large. In this limit it is shown that the density profile at (discrete) time $\nu$ is:

$$
n(x, \nu) \sim \exp \left(-\frac{x^{2}}{4 D \nu}\right) .
$$

Thus, the density profile is Gaussian, with a constant positive diffusion coefficient. The latter is

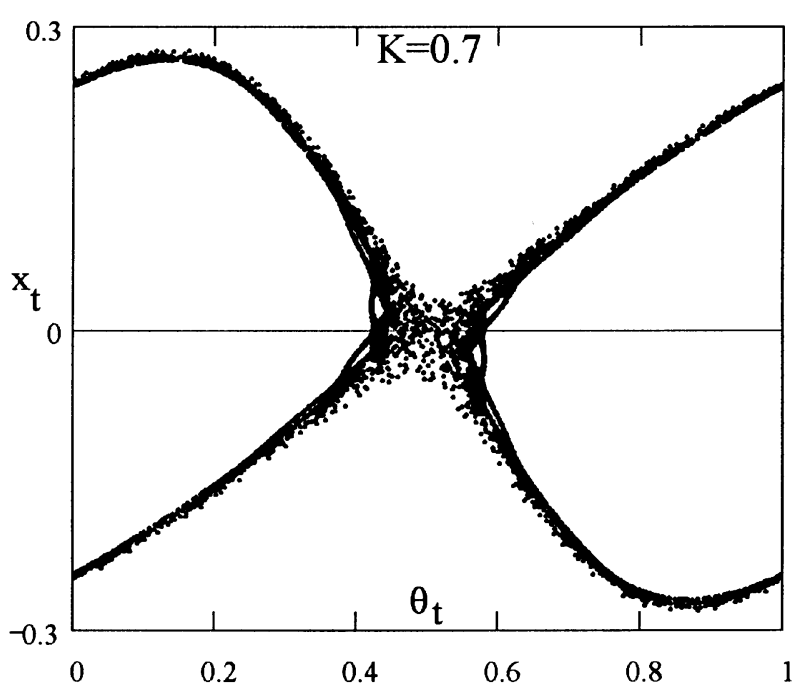

Figure 6. A chaotic orbit around the period-1 island in the standard map phase portrait. 


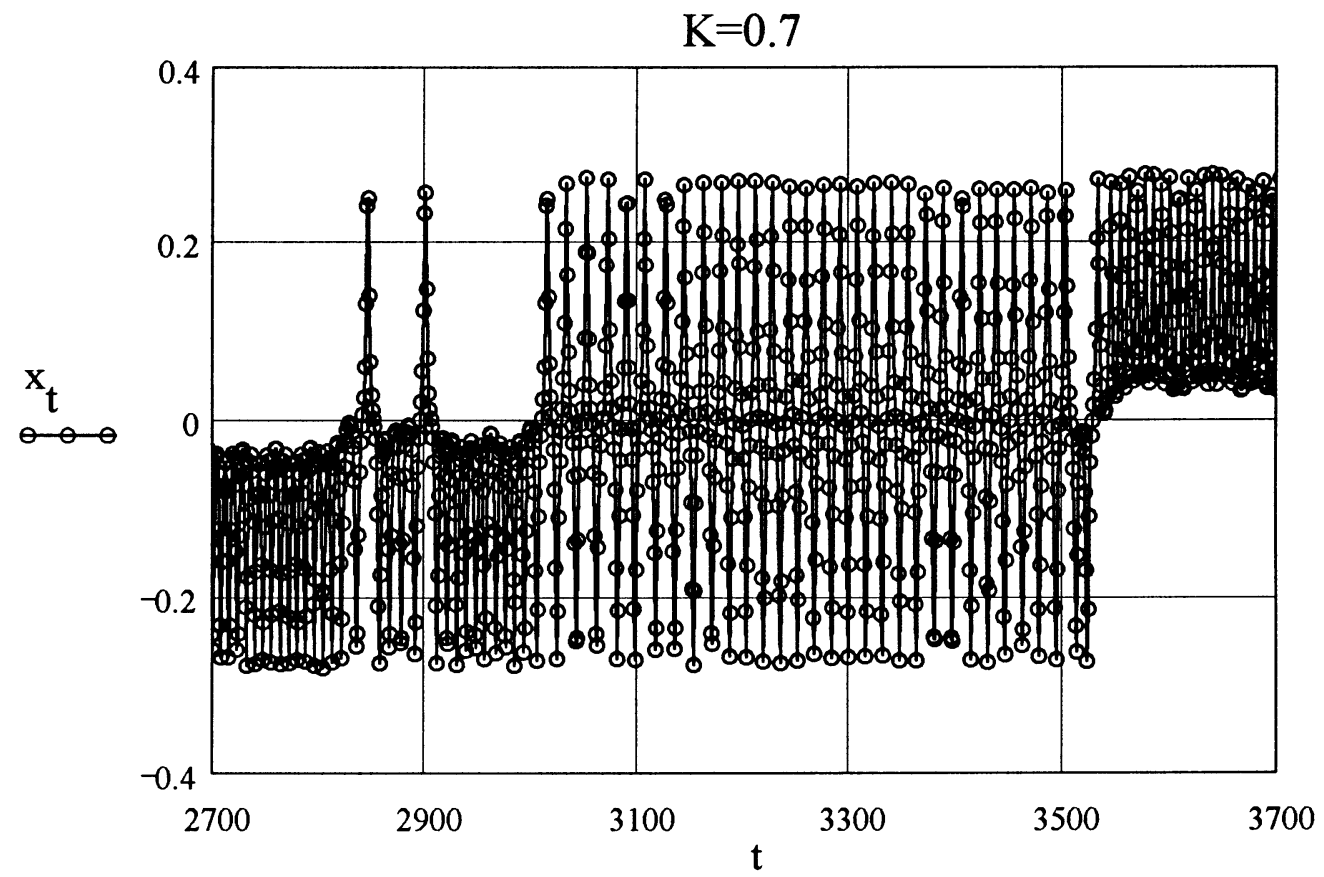

Figure 7. A section of the time series of the chaotic orbit of figure 6 .

given to the dominant ("quasilinear") order as follows:

$$
D_{\mathrm{QL}}=\frac{K^{2}}{4}
$$

It is clearly seen that now we deal with the typical situation of anomalous transport, in the sense defined in section 3. Indeed, the process has all the features of a diffusive process, but the diffusion coefficient is determined by $K$, a quantity that is not related to collisions. When the diffusion coefficient is evaluated to the next order in the small parameters (39), one finds:

$$
D=\frac{K^{2}}{4}\left[1-2 J_{2}(K)\right],
$$

where $J_{2}(K)$ is the Bessel function of order 2. The curious oscillations of this quantity as a function of $K$ (figure 5) may be due to the presence of islands, although this usual interpretation is not formally proven.

Numerical simulations confirm this behaviour of the diffusion coefficient up to a point: the position of the maxima is quite well reproduced, but the first maxima are much higher than indicated by (42). It has been shown that this behaviour is due to a specific property of the standard map: the variable $x$ is also periodic, as well as $\theta$ [this is easily seen in equations (38)]. As a result, there exist periodic orbits which wander indefinitely far both in the $\theta$ and $x$ directions: these are called accelerator modes [21]. Orbits starting in the neighbourhood of such a mode are carried away very far before decorrelating. It has been shown in detail that such a behaviour leads to a superdiffusive regime with $D \rightarrow \infty$ for certain ranges of $K$ 
(corresponding to the stability domains of the accelerator modes) [26], [27]. This explains quite well the presence of the peaks (singularities) in figure 5 .

A problem of great interest is the behaviour of chaotic orbits in the domain of an incomplete chaos, $K<K_{c}$. In particular, if we think of the diffusion of magnetic lines in a toroidal magnetic field configuration (as in a tokamak), the interest lies in situations in which the latter are effectively confined, and cannot wander out of the vessel. Consider a chaotic layer around an island chain, such as the one whose phase portrait is shown in figure 6 . The single chaotic orbit

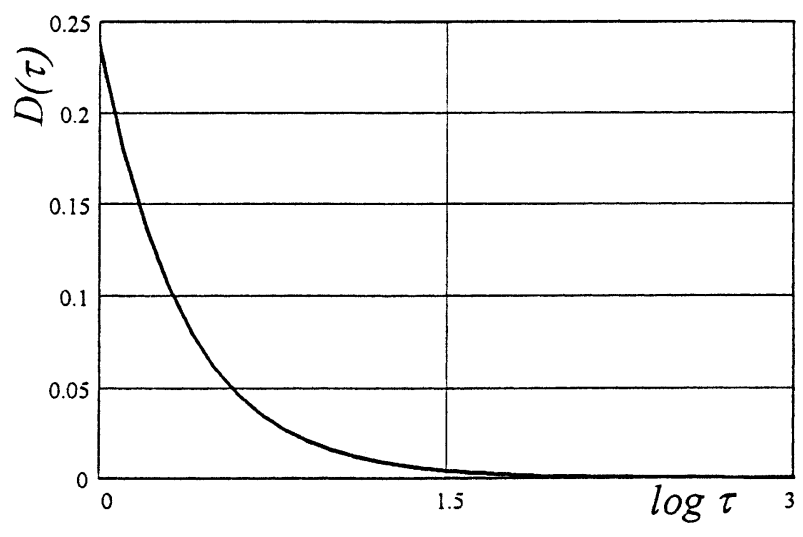

Figure 8. Running diffusion coefficient in the chaotic layer of figure 6 . shown there is sticking around the island. An alternative picture is obtained by showing the time series $x_{\nu}$ as a function of $\nu$ for a long orbit (figure 7 ). The particle has a very peculiar behaviour: for a certain time it oscillates pretty regularly, remaining all the time around the lower half of the island, then suddenly it jumps to another mode where it oscillates around the upper half, then it may jump to a mode where it oscillates around the whole island, etc. We thus identify three basins; the particle waits in one basin for an (apparently) random time, then jumps to another basin, etc. This behaviour strongly suggests a continuous time random walk $(C T R W)$ of the type studied in the previous section [28], [8], [29]. The adaptation of a CTRW to the present problem is very easily performed. The input, i.e. the transition probabilities $f_{n m}$ between the basins and the waiting time probabilities $p_{n}(t)$ in each basin are determined numerically by analyzing very long orbits [29]. A set of evolution equations for the density in the three basins is then derived and the mean square displacement, as well as the running diffusion coefficient are determined analytically from the latter. The result is a typical subdiffusive behaviour of the standard map in the subcritical regime $K<K_{c}$ characterized by a running diffusion coefficient (figure 8) which behaves asymptotically as:

$$
D_{\mathrm{SM}} \sim t^{-\beta}, \quad \beta \approx 1.1 .
$$

The subdiffusive behaviour is due, on the one hand, to the sticking of the orbits around the island and, on the other hand, to the presence of KAM barriers bounding the stochastic region and preventing the mean square displacement from growing indefinitely as in the case of a free diffusion. 


\section{Conclusion}

The main conclusion of this review is that the paradigm of the diffusive transport theory, based on the classical collisional kinetic theory and the theory of the Brownian motion has numerous and important exceptions. We insist on the fact that the signature (11) of strange transport, with $\alpha \neq 1$, is not a mere small quantitative difference from the diffusive regime. It rather implies a deep change of the evolution law, which becomes non-Markovian, hence, history-dependent. Whenever the memory function has a slow power-law decay in time, the standard Markovian model cannot even be considered as an approximation to the true evolution law.

Among the applications of the concept of strange transport, let us quote: fluid flow in disordered media (e.g., porous solids), percolative transport that introduces diffusion in a fractal environment, diffusion in a turbulent plasma, properties of polymers, etc. Clearly, a large number of fields of investigation open up for researchers in the domain of the transport theory, where D. N. Zubarev made pioneering contributions.

\section{References}

1. Zubarev D.N. Neravnovesnaya Statisticheskaya Termodinamika. Moscow, Nauka, 1971 (in Russian). [English translation: Nonequilibrium Statistical Thermodynamics. New York, Plenum Press, 1974.]

2. Boltzmann L. Weitere Studien über das Wärmegleichgewicht unter Gasmolekülen. Wien. Berichte, 1872, vol. 66, p. 275-370 (in German).

3. Bogoliubov N.N. Problemy Dinamicheskoi Teorii v Statisticheskoi Fizike. Moscow, 1946 (in Russian). [English translation: in Studies in Statistical Mechanics, edited by J. de Boer and G.E.Uhlenbeck, vol. 1, p. 5, Amsterdam, North Holland, 1962.]

4. Prigogine I. Nonequilibrium Statistical Mechanics. New York, Interscience, 1962. [Russian translation: Neravnovesnaya Statisticheskaya Mehanika. Moscow, Mir, 1964.]

5. Klimontovich Yu.L. Statisticheskaya Teoriya Neravnovesnykh Protsessov v Plazme. Moscow, 1964 (in Russian). [English translation: The Statistical Theory of Nonequilibrium Processes in a Plasma. Oxford, Pergamon, 1964.]

6. Balescu R. Equilibrium and Nonequilibrium Statistical Mechanics. New York, Wiley-Interscience, 1975. [Russian translation directed by D.N.Zubarev and Yu.L.Klimontovich, Ravnovesnaya i Neravnovesnaya Statisticheskaya Mehanika, 2 vols., Moscow, Mir, 1978.]

7. Balescu R. Transport Processes in Plasmas. 2 vols., Amsterdam, North Holland, 1988.

8. Balescu R. Statistical Dynamics: Matter out of Equilibrium. London, Imperial College Press, 1997.

9. Einstein A. Ueber die von der molekular-kinetischen Theorie der Wärme geforderte Bewegung von in ruhenden Flüssigkeiten suspendierten Teilchen. // Ann. d. Physik, 1905, vol. 17, p. 549-560 (in German).

10. Liewer P.C. Measurements of microturbulence in tokamaks and comparisons with theories of turbulent and anomalous transport. // Nucl. Fusion, 1985, vol. 25, No. 5, p. $543-621$. 
11. Shlesinger M.F., Zaslavsky G.M., Klafter J. Strange kinetics. // Nature, 1993, vol. 363, p. 31.

12. Gardiner C.W. Handbook of Stochastic Methods. 2nd ed., Berlin, Springer, 1997.

13. Balescu R., Wang H.-D., Misguich J. Langevin equation vs. kinetic equation: subdiffusive behaviour of charged particles in a stochastic magnetic field. // Phys. Plasmas, 1994, vol. 1, No. 12, p. 3826-3842.

14. Balescu R. // Phys. Rev. E, 1995, vol. 51, No. 5, p. 4807-4822.

15. Vanden Eijnden E., Balescu R. Transport in sheared stochastic magnetic fields. // Phys. Plasmas, 1997, vol. 4, No. 2, p. 270-276.

16. Montroll E.W., Weiss G.H. Random walks on lattices. II. // J. Math. Phys., 1965, vol. 6, No. 2, p. 167-181.

17. Montroll E.W., Shlesinger M.F. The wonderful world of random walks. - In: Studies in Statistical Mechanics (edited by J.L.Lebowitz and E.W.Montroll), vol. 11, p. 1-121, Amsterdam, North Holland, 1984.

18. Bouchaud J.P., Georges A. Anomalous diffusion in disordered media: statistical mechanisms, models and physical applications. // Phys. Rep., 1990, vol. 195, No. 4\&5, p. 127-293.

19. Weiss G.H. Aspects and Applications of Random Walks. Amsterdam, North Holland, 1994.

20. Chirikov B. A universal instability of many-dimensional oscillator systems. // Phys. Rep., 1979, vol. 52, No. 5, p. 265-379.

21. Lichtenberg A.J., Lieberman M.A. Regular and Stochastic Motion. Berlin, Springer, 1983.

22. Reichl L.E. The Transition to Chaos. Berlin, Springer, 1992.

23. Ott E. Chaos in Dynamical Systems. Cambridge, Cambridge Univ. Press, 1993.

24. Rechester A.B., White R.B. Calculation of turbulent diffusion for the Chirikov-Taylor model. // Phys. Rev. Lett., 1980, vol. 44, No. 24, p. 1586-1589.

25. Hasegawa H.H., Saphir W.C. - In: Aspects of Nonlinear Dynamics (edited by I.Antoniou and F.Lambert), Berlin, Springer, 1991.

26. Ichikawa Y., Kamimura T., Hatori T. // Physica D, 1987, vol. 29, p. 247-255.

27. Horita T., Hata H., Ishizaki R., Mori H. // Prog. Theor. Phys., 1990, vol. 83, No. 6, p. $1065-1070$.

28. Balescu R. Continuous time random walk model for standard map dynamics. // Phys. Rev. E, 1997, vol. 55, No. 3, p. 2465-2474.

29. Misguich J., Reuss J.D., Elskens Y., Balescu R. Motion in a stochastic layer described by symbolic dynamics. // Chaos, 1998 (to be published). 


\section{Аномальна дифузія}

\section{Р.Балеску}

Асоціація “Євроатом - Бельгія" з термоядерного синтезу,

Вільний університет м. Брюселя, СР 231,

Кампус Плен, бульвар Перемоги,

1050 м. Брюсель, Бельгія

Отримано 25 березня 1998 р.

Подається означення аномальної дифузії як процесу, при якому середнє квадратичне зміщення випадкового блукання частинки, що розглядається, має асимптотичну поведінку $\left\langle\boldsymbol{x}^{2}(t)\right\rangle \sim \boldsymbol{t}^{\alpha}$, де $\alpha \neq 1$. Подається короткий огляд властивостей таких процесів, особливо наголошується на глибокій різниці з відповідною нормальною дифузійною еволюцією. Обговорюється багато прикладів, у тому числі дифузія заряджених частинок у флуктуюючому магнітному полі, неперервні часові випадкові блукання та процеси переносу в системах з хаотичним гамільтоніаном.

Ключові слова: аномальна дифузія, випадковий процес, хаос

PACS: $05.40 .+j, 05.45 .+b, 83.50 . W$ 
